\title{
c-Abl inhibition mitigates diet-induced obesity through improving insulin sensitivity of subcutaneous fat in mice
}

\author{
Rong Wu ${ }^{1,2}$. Jian-guang Sun ${ }^{1,3} \cdot$ Ji-qiu Wang ${ }^{4} \cdot{\text { Binhua } \mathrm{Li}^{5}}^{5}$ Qingsong Liu ${ }^{5}$. \\ Guang Ning ${ }^{4}$ - Wanzhu Jin ${ }^{6}$ - Zengqiang Yuan ${ }^{1,2,7}$
}

Received: 30 October 2016 / Accepted: 14 December 2016 / Published online: 10 January 2017

(C) Springer-Verlag Berlin Heidelberg 2017

\begin{abstract}
Aims/hypothesis High-energy diets are among the main causes of the global epidemic of metabolic disorders, including obesity and type 2 diabetes. The mechanisms of highenergy-diet-induced metabolic disorders are complex and largely unknown. The non-receptor tyrosine kinase c-Abl plays an important role in adipogenesis in vitro but its role in vivo in the regulation of metabolism is still elusive. Hence,
\end{abstract}

Rong $\mathrm{Wu}$ and Jian-guang Sun contributed equally to this work.

Electronic supplementary material The online version of this article (doi:10.1007/s00125-016-4202-2) contains peer-reviewed but unedited supplementary material, which is available to authorised users.

Wanzhu Jin

jinw@ioz.ac.cn

$\triangle$ Zengqiang Yuan

zqyuan@ibp.ac.cn

1 State Key Laboratory of Brain and Cognitive Sciences, Institute of Biophysics, Chinese Academy of Sciences, 15 Datun Road, Chaoyang District, Beijing 100101, China

2 College of Life Sciences, University of Chinese Academy of Sciences, Beijing, China

3 Sino-Danish Center Neuroscience Program, University of Chinese Academy of Sciences, Beijing, China

4 Ruijin Hospital Affiliated to Shanghai Jiaotong University School of Medicine, Shanghai, China

5 High Magnetic Field Laboratory, Chinese Academy of Sciences, Hefei, Anhui, China

6 Key Laboratory of Animal Ecology and Conservation Biology, Institute of Zoology, Chinese Academy of Sciences, 1 Beichen West Road, Chaoyang District, Beijing 100101, China

7 Center of Alzheimer's Disease, Beijing Institute for Brain Disorders, Beijing, China we sought to address the role of c-Abl in diet-induced obesity and obesity-associated insulin resistance.

Methods The expression of c-Abl in different fat tissues from obese humans or mice fed a high-fat diet (HFD) were first analysed by western blotting and quantitative PCR. We employed conditional deletion of the $c-A b l$ gene (also known as Abll) in adipose tissue using Fabp4-Cre and 6-week-old mice were fed with either a chow diet (CD) or an HFD. Age-matched wild-type mice were treated with the c-Abl inhibitor nilotinib or with vehicle and exposed to either CD or HFD, followed by analysis of body mass, fat mass, glucose and insulin tolerance. Histological staining, ELISA and biochemical analysis were used to clarify details of changes in physiology and molecular signalling.

Results c-Abl was highly expressed in subcutaneous fat from obese humans and HFD-induced obese mice. Conditional knockout of $c-A b l$ in adipose tissue improved insulin sensitivity and mitigated HFD-induced body mass gain, hyperglycaemia and hyperinsulinaemia. Consistently, treatment with nilotinib significantly reduced fat mass and improved insulin sensitivity in HFD-fed mice. Further biochemical analyses suggested that c-Abl inhibition improved whole-body insulin sensitivity by reducing HFD-triggered insulin resistance and increasing adiponectin in subcutaneous fat.

Conclusions/interpretation Our findings define a new biological role for c-Abl in the regulation of diet-induced obesity through improving insulin sensitivity of subcutaneous fat. This suggests it may become a novel therapeutic target in the treatment of metabolic disorders.

Keywords Adiponectin · c-Abl $\cdot$ Insulin resistance · Nilotinib · Obesity 


$\begin{array}{ll}\text { Abbreviations } \\ \text { BAT } & \text { Brown adipose tissue } \\ \text { CD } & \text { Chow diet } \\ \text { cKO } & \text { Conditional knockout } \\ \text { CML } & \text { Chronic myelogenous leukaemia } \\ \text { ER } & \text { Endoplasmic reticulum } \\ \text { eWAT } & \text { Epidymal white adipose tissue } \\ \text { GLM } & \text { General linear model } \\ \text { HFD } & \text { High-fat diet } \\ \text { ITT } & \text { Insulin tolerance test } \\ \text { JNK } & \text { c-Jun amino-terminal kinase } \\ \text { PDGFR } & \text { Platelet-derived growth factor receptor } \\ \text { PI3K } & \text { Phosphatidylinositol 3-kinase } \\ \text { RBP-4 } & \text { Retinol-binding protein 4 } \\ \text { sWAT } & \text { Subcutaneous white adipose tissue } \\ \text { WT } & \text { Wild-type }\end{array}$

\section{Introduction}

Obesity and type 2 diabetes have become a global pandemic and are among the main causes of morbidity and mortality [1, 2]. Energy-dense food and a sedentary western lifestyle result in a surplus of energy stored in the adipose tissue, leading to the development of obesity and metabolic disorders [3]. In these metabolic disorders, tissues such as muscle, liver and fat become less responsive or even resistant to insulin [4].

Insulin resistance is a fundamental pathogenic factor shared by a myriad of metabolic disorders including obesity and type 2 diabetes [5]. Upon consumption of a high-fat diet (HFD), multiple factors contribute to insulin resistance, including NEFA, hormones and inflammation [6]. The distribution of body fat is also a critical determinant of insulin sensitivity. Lean individuals with a mainly subcutaneous distribution of fat are more insulin sensitive than lean individuals who have more visceral fat [7]. Differences in the characteristics of adipose tissue from these two depots might explain in part why the metabolic effects are different in visceral and subcutaneous fat. Visceral fat expresses more genes encoding secretory proteins, such as resistin and retinol-binding protein 4 (RBP4), which are responsible for insulin resistance. Subcutaneous fat releases more adiponectin than visceral fat [8].

The non-receptor tyrosine kinase c-Abl is ubiquitously expressed and mediates multiple signalling cascades governing the cell cycle and cell adhesion, proliferation and apoptosis [9-12]. Various c-Abl inhibitors have been used to treat chronic myelogenous leukaemia (CML) [13]. One such drug, imatinib, was used to control CML through targeting the Bcr-Abl fusion protein [14-16]. Interestingly, imatinib treatment induced a significant regression in type 2 diabetes in CML patients [17]. Consistently, two recent studies showed that imatinib improved insulin sensitivity in the peripheral tissues via increasing plasm adiponectin levels $[18,19]$. In addition, imatinib was also found to increase the survival of insulin-producing beta cells via induction of phosphatidylinositol 3-kinase signalling or promotion of $N K X 2.2$ (also known as $N K X 2-2$ ) and GLUT-2 (also known as $S L C 2 A 2)$ gene expression [20, 21]. A recent study indicated that imatinib regulates insulin homeostasis through inhibiting the platelet-derived growth factor receptor (PDGFR), which is also a target of imatinib [22].

A proteomic analysis revealed that $\mathrm{c}-\mathrm{Abl}$ was a potential key regulator of adipogenesis via regulation of the expression and activity of peroxisome proliferator-activator receptor $\gamma 2$ (PPAR $\gamma 2$ ), the master adipogenic regulator controlling adipogenesis $[23,24]$. These findings indicate that $\mathrm{c}-\mathrm{Abl}$ participates in adipocytes metabolism regulation in vitro. However, the in vivo role of $\mathrm{c}-\mathrm{Abl}$ in adipose tissue and whether $\mathrm{c}-\mathrm{Abl}$ inhibition can improve obesity-associated insulin sensitivity remains to be further explored.

Studies based on genetic models and/or specific c-Abl kinase inhibitors are urgently needed to confirm the role of c-Abl in insulin homeostasis. In this study, we employed a conditional ablation of c-Abl in adipose tissue and a specific inhibitor to test our hypothesis that c-Abl plays an important role in dietinduced obesity and obesity-associated insulin resistance.

\section{Methods}

Animals All mice used had C57BL/6J background. Mice were housed at $22-24^{\circ} \mathrm{C}$ under a $12 \mathrm{~h}$ light-dark cycle and had free access to water. All mice were maintained in the Animal Care Facility at the Institute of Biophysics, Chinese Academy of Sciences, Beijing. Six-week-old mice were randomised to feeding with chow diet (CD) or HFD (60\% of total energy from fat). All the experiments were blind to group assignment and outcome assessment.

All experiments involving animals were approved by and conformed to the guidelines of the institutional animal care and ethics committee at the Institute of Biophysics of the Chinese Academy of Sciences (Beijing, China).

Generation of mice with a conditional knockout of $c-A b l$ in adipose tissue $c-A b l^{\text {flox/flox }}$ mice (a gift from Y. Cang, Zhejiang University, Hanzhou, China; $c-A b l$ is also known as $A b l l)$ were crossed with Fabp4-Cre mice (a gift from W. Jin, Institute of Zoology, Chinese Academy of Sciences, Beijing, China). The offspring male $c-A b l^{\text {flox/flox }:: F a b p 4-C r e}{ }^{+/-}(c-A b l$ conditional knockout $[\mathrm{cKO}]$ ) mice and $c-A b t^{\text {flox/flox }}$ (wild-type [WT]) mice were used in the experiments.

GTTs and insulin tolerance tests After being fasted for $16 \mathrm{~h}$ and $4 \mathrm{~h}$, respectively, mice were subjected to GTTs and insulin tolerance tests (ITTs) (see ESM Methods for details). 
Energy intake, expenditure and activity measurements See ESM Methods for details of metabolic measurements made in mice.

Nilotinib treatment Six-week-old C57BL/6J male mice upon CD or HFD were randomised to receive orally administered nilotinib $(75 \mathrm{mg} / \mathrm{kg})$ or vehicle at $48 \mathrm{~h}$ intervals for 16 weeks.

Histological analysis Mice were fasted for $16 \mathrm{~h}$, anaesthetised with ether and then killed before blood and tissue collection. Metabolism-related tissues were stained with haematoxylin and eosin (see ESM Methods for details).

ELISA Serum insulin and adipokines were measured by ELISA (see ESM Methods for details).

Real-time quantitative RT-PCR Real-time quantitative RT-PCR was used to determine relative gene expression levels. After treatments, tissues, including adipose tissue, liver and muscle, were dissected and total RNA was extracted. For further details, see ESM Methods. Tbp was used as a housekeeping gene used for input normalisation. Detected genes and their primers are shown in ESM Table 1.

Immunoblotting Tissues were dissected, lysed in lysis buffer and subjected to western blotting and protein quantification. The proteins were detected with antibodies against c-Abl, phospho-cAbl, Akt, phospho-Akt, c-Jun amino-terminal kinase (JNK), phospho-JNK, Grp78, GAPDH and $\beta$-tubulin. For details, see ESM Methods. The intensity of the western blot bands was determined using ImageJ software (NIH, Bethesda, MD, USA).

Human study Visceral and subcutaneous fat was obtained from patients at Ruijin Hospital, Shanghai Jiao Tong University School of Medicine, for determination of c-Abl protein levels in adipose tissue. The adipose tissues from obese individuals $\left(\mathrm{BMI}>30 \mathrm{~kg} / \mathrm{m}^{2}\right.$ ) were obtained from hospitalised individuals undergoing weight-loss surgery. The control adipose tissues from matched normal-weight individuals $\left(\mathrm{BMI}<30 \mathrm{~kg} / \mathrm{m}^{2}\right)$ were obtained from hospitalised patients undergoing surgery for gallstones or hernia, without fever and other critical conditions. All individuals were from the eastern area of China. Details of patients are given in ESM Table 2. The adipose issues were subjected to immunoblotting with c-Abl antibody. This study was approved by the Institutional Review Board of the Ruijin Hospital, Shanghai Jiao Tong University School of Medicine, and was in accordance with the principle of the Helsinki Declaration II. Written informed consent was obtained from each participant.

Statistical analysis Statistical analyses were performed by ANOVA followed by Tukey's post hoc test or by a two-tailed Student's $t$ test. All values are expressed as means \pm SEM.
$* p<0.05, * * p<0.01$ and $* * * p<0.001$ denote the significance thresholds. There was no exclusion of any data, samples or animals.

\section{Results}

c-Abl expression is increased in subcutaneous white adipose tissue from obese humans and HFD-induced obese mice Despite previous studies showing that c-Abl was activated during adipogenesis, the expression of $\mathrm{c}-\mathrm{Abl}$ in obese human and mouse adipose tissue has never been investigated. Here, we found that the protein levels of c-Abl were markedly increased in subcutaneous fat from obese humans. However, there was no significant difference in visceral fat (Fig. 1a,b). Interestingly, the protein levels of c-Abl in subcutaneous fat were positively related to BMI (Fig. 1c and ESM Table1). a

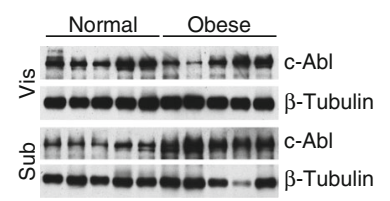

C

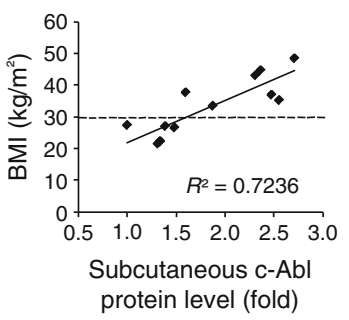

$\mathbf{e}$

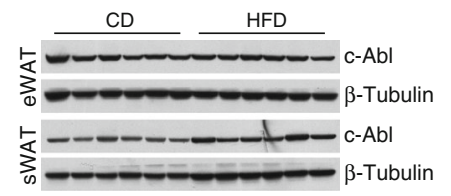

b

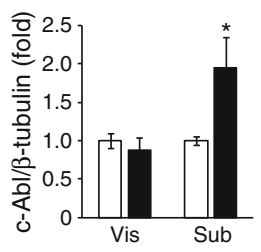

d
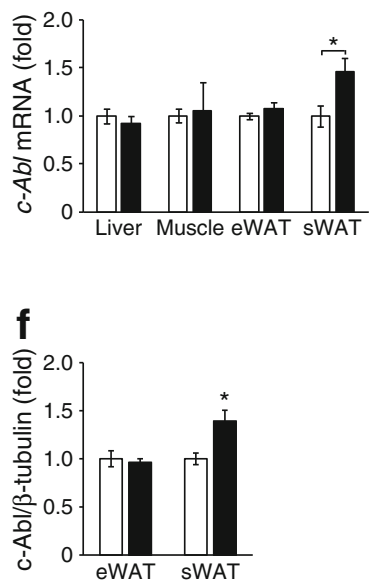

Fig. 1 c-Abl expression is increased in sWAT from obese humans and HFD-induced obese mice. (a, b) Representative immunoblots of lysates of visceral (Vis) and subcutaneous (Sub) fat from normal or obese humans with the antibodies indicated (a) and the level of c-Abl normalised to $\beta$-tubulin (b). Data are means $\pm \operatorname{SEM}$ (normal $n=6$, obese $n=7$ ). White bars, normal; black bars, obese. ${ }^{*} p<0.05$ vs normal (Student's $t$ test). (c) The relative protein levels of c-Abl in human subcutaneous fat were positively related to BMI. (d) The mRNA levels of $c$ - $A b l$ in different tissues from mice fed with CD or HFD ( $n=6$ per group). White bars, CD; black bars, HFD. $* p<0.05$ vs CD (Student's $t$ test). (e, f) Representative immunoblots of lysates of eWAT and sWAT from CD-fed mice and HFDinduced obese mice with the antibodies indicated (e) and the levels of cAbl normalised to $\beta$-tubulin (f). Data are expressed as means \pm SEM ( $n=6$ per group). White bars, CD; black bars, HFD. ${ }^{*} p<0.05$ vs CD (Student's $t$ test) 
Similar findings were observed in HFD-induced obese mice. The mRNA levels of $c-A b l$ increased in subcutaneous white adipose tissue (sWAT) (Fig. 1d). Immunoblotting also showed an increased c-Abl protein level in sWAT but no significant difference in epidymal white adipose tissue (eWAT) (Fig. 1e,f). Together, these results indicated that subcutaneous c-Abl protein levels are co-related with obesity.

cKO of $c-A b l$ in adipose tissue protects against HFD-induced obesity Our finding that c-Abl was selectively increased in obese human and mouse subcutaneous fat led us to examine whether $\mathrm{CKO}$ of $c-A b l$ in adipose tissue protects against HFDinduced obesity. Fabp4-Cre has been widely used to target adipose tissue $[25,26]$. We crossed $c-A b l^{\text {flox/flox }}$ mice with Fabp4-Cre mice to generate offspring. c-Abl cKO mice $\left(c-A b t^{\left.\text {flox/flox }:: F a b p 4-\mathrm{Cre}^{+/-}\right)}\right.$and WT mice $\left(c-A b f^{\text {flox/flox }}\right)$ were fed with HFD or CD. Interestingly, Fabp4-Cre-mediated $c-A b l$ deletion in adipose tissue significantly decreased c-Abl protein level only in sWAT and not in other adipose tissues (ESM Fig. 1a-c). Therefore, Fabp4-Cre mediated $c-A b l$ deletion provides an ideal model for studying the role of c-Abl in subcutaneous fat. We found that Fabp4-Cre mediated $c-A b l \mathrm{cKO}$ remarkably reduced HFD-induced body mass gain, while no change was observed in mice on CD (Fig. 2a). MRI analysis showed that a HFD significantly induced lean and fat mass gain in mice, while $c-A b l \mathrm{cKO}$ significantly reduced these gains (Fig. 2b,c). Interestingly, $c-A b l \mathrm{cKO}$ had no effect on the lean or fat mass in CD-fed mice, indicating that Fabp4-Cre mediated $c-A b l$ inactivation did not cause developmental defects (Fig. $2 b, c)$. Similarly, specific $c-A b l$ deletion in adipose tissue reduced the mass of sWAT, brown adipose tissue (BAT) and liver, but not eWAT, under HFD feeding conditions (Fig. 2d-g); there was no significant change under CD (Fig. 2d-g). Histological analysis revealed that $c-A b l$ conditional deletion in adipose tissue markedly attenuated HFD-induced adipocyte hypertrophy and hepatosteatosis (Fig. 2h). Interestingly, there was no significant change in the size of adipocytes from eWAT when comparing WT and c-Abl cKO mice under HFD (Fig. 2h). Taken together, these results indicate that specific deletion of $c-A b l$ in adipose tissue protects against HFDinduced obesity.

Since the specific $c-A b l$ deletion in adipose tissue significantly reduced lipid accumulation in BAT (Fig. 2h), we wondered whether $\mathrm{cKO}$ of $c-A b l$ could protect mice against HFDinduced obesity through upregulation of energy expenditure. Activated BAT increases the consumption of fatty acids and glucose, which leads to increased energy expenditure [27]. However, we found that there was no significant difference in BAT levels of thermogenesis-related gene $U c p 1$ and fatty acid oxidation-related genes between WT and KO mice (Fig. 3a). Additionally, there were no significant changes in oxygen consumption relative to lean mass under either CD or HFD condition (Fig. 3b-d). According to the standard protocol of energy

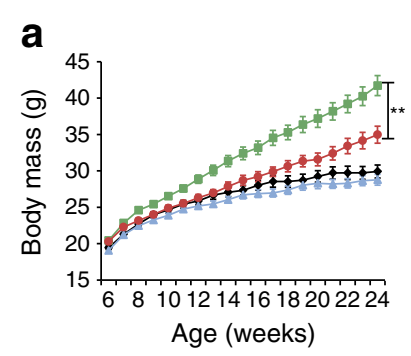

b
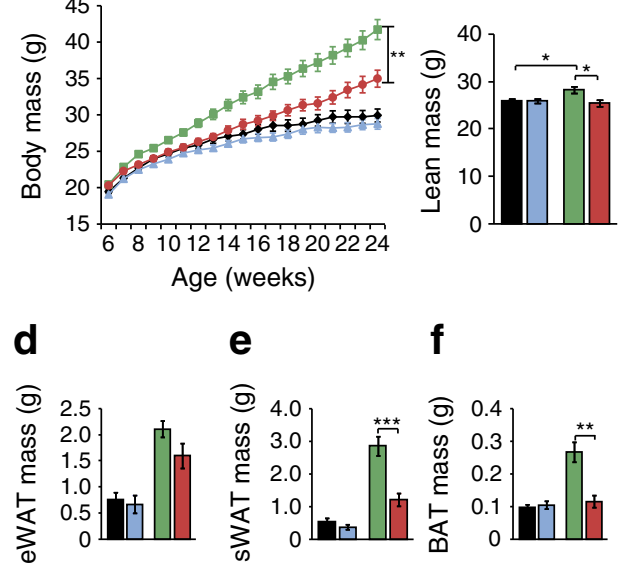

f

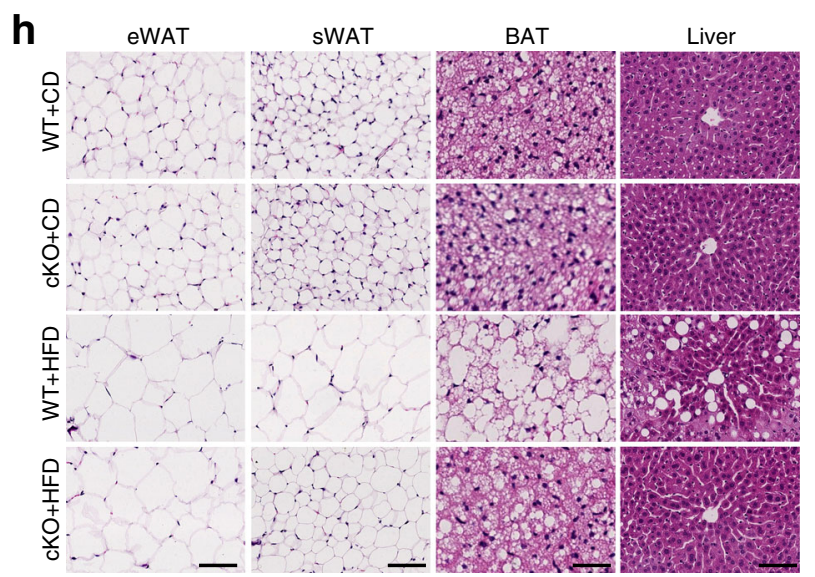

Fig. 2 Conditional deletion of $c-A b l$ in adipose tissue protects against HFD-induced obesity in mice. (a) Body mass of WT and $c-A b l^{\text {flox }}$ flox::Fabp4-Cre (cKO) male mice fed with CD or HFD. WT+CD, $n=21$; cKO+CD, $n=17$; WT+HFD, $n=26$; cKO+HFD, $n=20$. (b, c) Lean and fat mass measured by MRI. WT+CD, $n=10$; cKO+CD, $n=9$; WT+HFD, $n=10$; cKO+HFD, $n=9$. (d-g) Mass of eWAT (d), $\operatorname{sWAT}(\mathbf{e})$, BAT (f) and liver (g). WT+CD, $n=15 ; \mathrm{cKO}+\mathrm{CD}, n=9$; WT+HFD, $n=17$; cKO+HFD, $n=10$. In graphs, data are means \pm SEM. Black bars and diamonds, $\mathrm{WT}+\mathrm{CD}$; blue bars and triangles, $\mathrm{cKO}+\mathrm{CD}$; green bars and squares, WT+HFD; red bars and circles, cKO+HFD. ${ }^{*} p<0.05$, $* * p<0.01$ and $* * * p<0.001$ for indicated comparisons (ANOVA). (h) Haematoxylin and eosin staining of eWAT, sWAT, BAT and liver. Scale bar, $100 \mu \mathrm{m}$ (eWAT, sWAT, liver) or $50 \mu \mathrm{m}$ (BAT)

expenditure analysis between groups with different body mass $[28,29]$, we performed a stringent statistical test (general linear model [GLM] and ANCOVA). We found that $c-A b l \mathrm{cKO}$ in adipocytes also had no significant effect on energy expenditure under either HFD or CD condition (Fig. 3e,f). Furthermore, there was no phenotypic difference in locomotive ability between these mice (Fig. 3g). We next sought to determine whether specific deletion of $c-A b l$ in adipose tissue reduced food intake. Interestingly, $c-A b l$ cKO had no effect on food intake under $\mathrm{CD}$ but slightly increased the food intake relative to body mass under HFD condition (Fig. 3h). Furthermore, under HFD treatment, the serum leptin level was significantly higher in WT mice and this level was reduced by $c-A b l$ cKO (Fig. 3i). Taken 


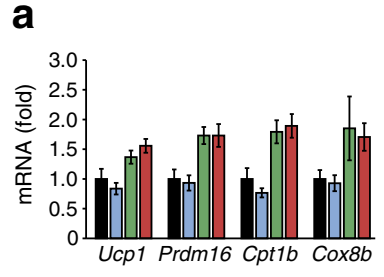

b
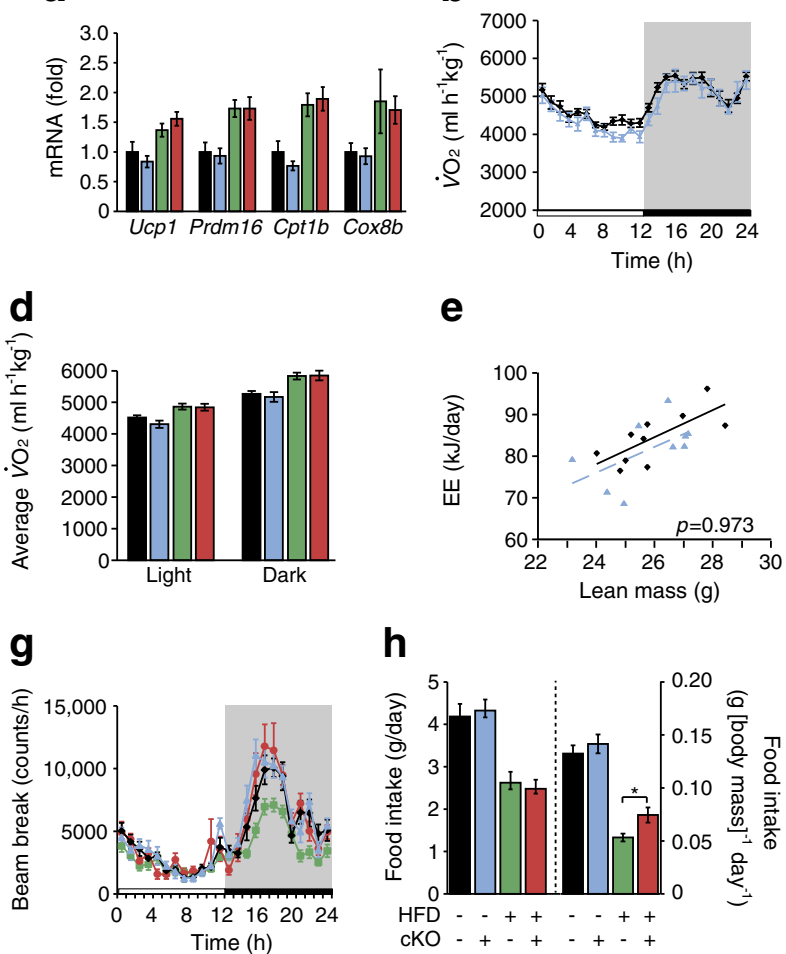

C

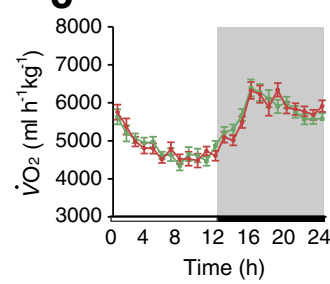

f

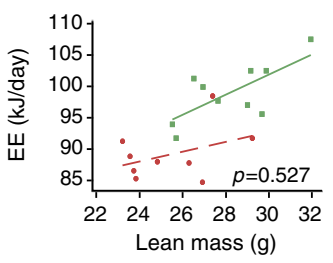

i

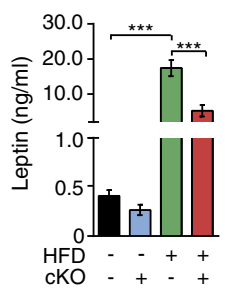

Fig. 3 Adipose-specific deletion of $c-A b l$ has no effect on energy balance in obese mice. (a) Relative gene expression in BAT from indicated groups ( $n=5$ per group). (b-d) Oxygen consumption of WT and $c-A b l$ cKO male mice fed with CD (b) or HFD (c) and average oxygen consumption during light and dark period (d). WT $+\mathrm{CD}$, $n=10 ; \mathrm{cKO}+\mathrm{CD}, n=9 ; \mathrm{WT}+\mathrm{HFD}, n=10 ; \mathrm{cKO}+\mathrm{HFD}, n=9$. (e, f) Statistical analysis by GLM and ANCOVA of the energy expenditure (EE) of $c-A b l \mathrm{cKO}$ and WT mice fed with CD (e) or HFD (f). (g) Locomotive activity of $c-A b l \mathrm{cKO}$ and WT mice fed with CD or HFD.

together, specific deletion of $c-A b l$ in adipose tissue protects against HFD-induced obesity and this does not occur through reduction of energy intake.

Deletion of $\boldsymbol{c}-\boldsymbol{A} \boldsymbol{b l}$ in adipocytes protects against HFDinduced insulin resistance To define the relationship between whole-body insulin sensitivity and the development of obesity, we performed GTTs and ITTs at different time points after dietary intervention. As expected, the HFD led to glucose intolerance and insulin resistance in WT mice at 15 weeks old (Fig. 4a,c), and both glucose intolerance and insulin resistance were progressively exacerbated in WT mice following HFD treatment (Fig. 4b,d). At week 24 following HFD treatment, the WT mice developed hyperglycaemia, hyperinsulinaemia and compensatory hypertrophy of pancreatic islets (Fig. $4 \mathrm{e}-\mathrm{g}$ ). Compared with WT mice, $c-A b l$ cKO mice showed significant mitigation of the HFD-induced phenotypes and improved insulin sensitivity during HFD (Fig. 4a-f). In addition, HFD-induced pancreatic hypertrophy was also markedly ameliorated in $c$-Abl cKO mice (Fig. 4g).
$\mathrm{WT}+\mathrm{CD}, n=11 ; \mathrm{cKO}+\mathrm{CD}, n=9 ; \mathrm{WT}+\mathrm{HFD}, n=12 ; \mathrm{cKO}+\mathrm{HFD}, n=12$ (h) Daily food intake in absolute values and normalised by body mass. WT+CD, $n=10$; cKO+CD, $n=9$; WT+HFD, $n=10$; cKO+HFD, $n=9$. (i) Leptin levels in serum from the indicated group of mice. WT+CD, $n=6$; $\mathrm{cKO}+\mathrm{CD}, n=4$; WT+HFD, $n=16$; cKO+HFD, $n=10$. For graphs, data are means \pm SEM, except for (e) and (f). Black bars and diamonds, WT+CD; blue bars and triangles, $\mathrm{CKO}+\mathrm{CD}$; green bars and squares, WT+HFD; red bars and circles, cKO++HFD. $* p<0.05$ and $* * * p<0.001$ for indicated comparisons (ANOVA)

Insulin sensitivity has been reported to be closely related to phosphatidylinositol 3-kinase (PI3K)-Akt signalling. Upon insulin stimulation, the intrinsic tyrosine kinase of the insulin receptor leads to receptor autophosphorylation at multiple tyrosine residues and the phosphorylation of the insulin receptor substrates (IRS1/2) leads to the activation of PI3K-Akt signalling pathway and regulation of glucose homeostasis [4]. Hence, we further explored whether $c-A b l$ conditional deletion altered the levels of phosphorylated Akt in adipose tissue. Immunoblotting analysis revealed that insulin treatment obviously increased Akt phosphorylation in adipose tissue and that HFD treatment significantly reduced the Akt phosphorylation (Fig. 4h). Interestingly, we found that Fabp4-Cre mediated c$\mathrm{Abl}$ deletion reduced c-Abl expression and rescued Akt phosphorylation in sWAT but not in eWAT or BAT (Fig. 4h and ESM Fig. 1a-c). Collectively, cKO of $c-A b l$ in adipocytes protects mice against HFD-induced insulin resistance.

Nilotinib, a specific c-Abl inhibitor, protects against HFDinduced obesity We wanted to test whether the above effect of c-Abl in obesity development was dependent on its kinase 
Fig. 4 cKO of $c-A b l$ improves whole-body insulin sensitivity. (a, b) GTT results for WT and c-Abl cKO mice fed with $\mathrm{CD}$ or HFD at 15 weeks (a) and 24 weeks (b) of age ( $n=6-8$ per group). The AUC for the GTT is also shown. (c, d) ITT results for WT and $c-A b l \mathrm{cKO}$ mice fed with CD or HFD at 16 weeks (c) and 25 weeks (d) of age ( $n=6-8$ per group). The AUC for the ITT is also shown. (e, f) Blood glucose $(\mathbf{e}, n=8)$ and insulin levels (f, $n=5-9)$, in the fasted state, of WT and $c-A b l \mathrm{cKO}$ mice fed with CD or HFD for 24 weeks. (g) Haematoxylin and eosin staining of pancreases from WT and $c-A b l$ cKO mice fed with CD or HFD for 24 weeks. Scale bar, $200 \mu \mathrm{m}$. (h) Immunoblot of lysates from indicated tissues with the antibodies indicated.

Quantification of phosphorylated Akt in the indicated tissues is shown. For graphs, data are means \pm SEM. Black bars and diamonds, WT+CD; blue bars and triangles, $\mathrm{cKO}+\mathrm{CD}$; green bars and squares, WT+HFD; red bars and circles, cKO+HFD. $* p<0.05, * * p<0.01$ and $* * * p<0.001$ for indicated comparisons (ANOVA). In the line graphs in $(\mathbf{a}-\mathbf{d}), p$ values are for red circles vs green squares at the indicated time points
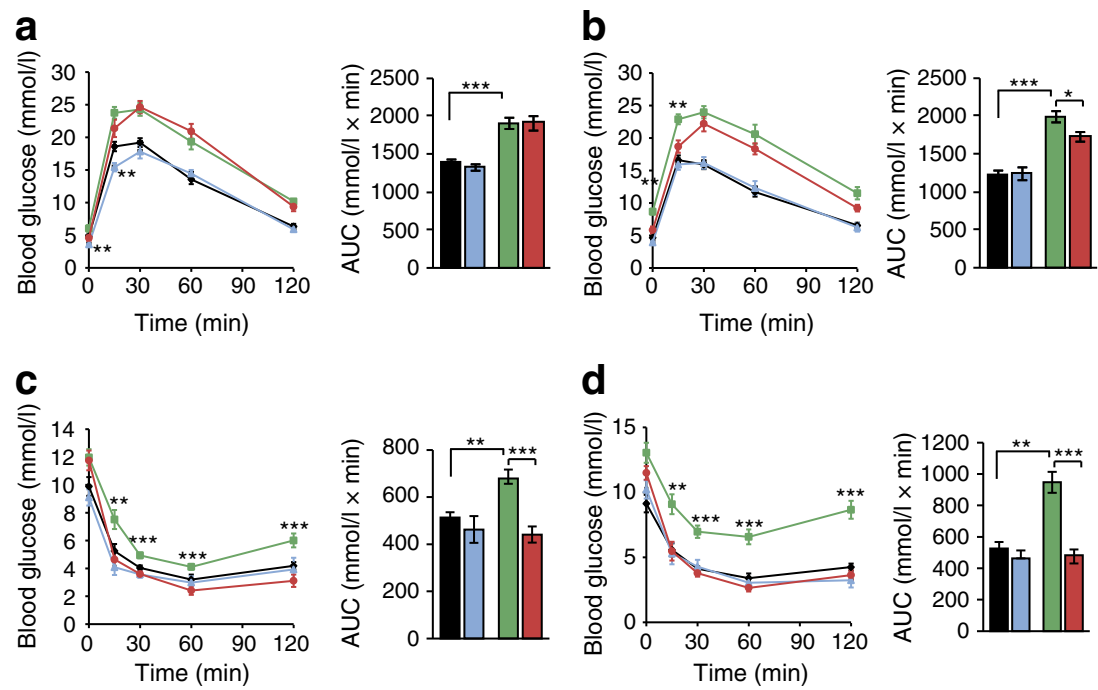

d
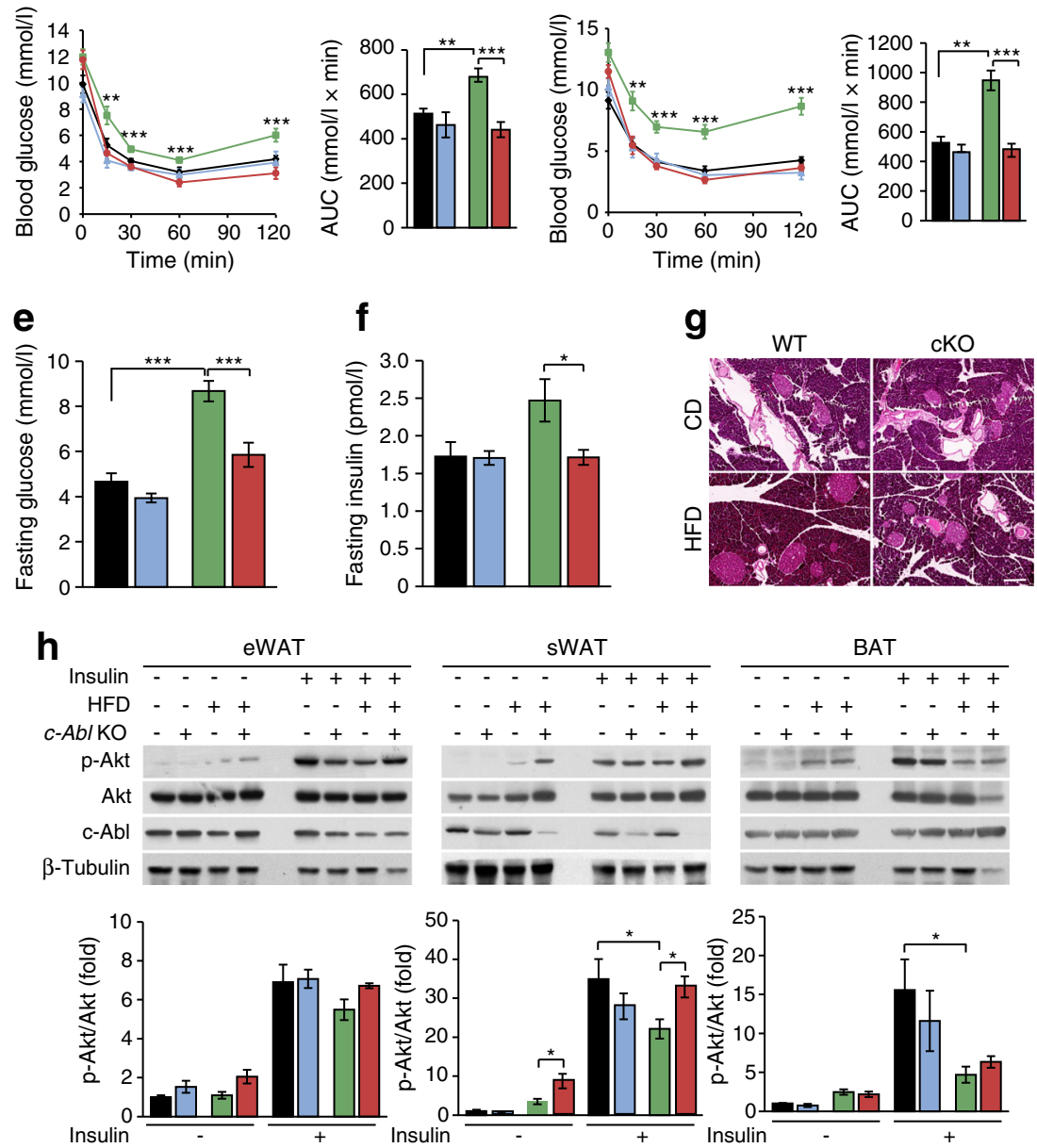

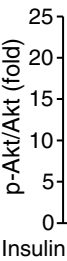

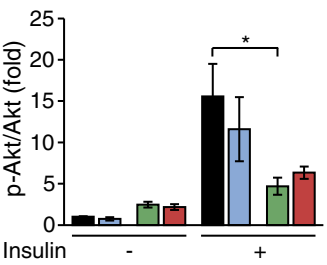

activity and to gauge the potential therapeutic effect of c-Abl inhibition on metabolic disorders. To do this, we administered nilotinib, a c-Abl kinase inhibitor, orally at $48 \mathrm{~h}$ intervals to HFD-induced obese mice. We found that nilotinib treatment significantly reduced HFD-induced body mass gain (Fig. 5a). MRI analysis showed that nilotinib treatment decreased fat mass in both CD- and HFD-fed mice (Fig. 5c), while it has no effect on the lean mass (Fig. 5b). Further examination showed that nilotinib treatment led to a remarkable decrease in the mass of all fat tissues including eWAT, sWAT and BAT (Fig. 5d-g). Histological analysis revealed that lipid droplets in eWAT, sWAT, BAT and liver from nilotinib-treated mice were smaller than those in tissues from control mice under HFD treatment (Fig. 5h). As with $c-A b l$ cKO, nilotinib treatment failed to alter oxygen consumption and energy expenditure in either HFD- or CD-fed mice (Fig. 5i-1). Interestingly, nilotinib treatment reduced the body mass (Fig. 5a) despite an increase in food intake under the HFD condition (Fig. 5m). Nilotinib treatment also reduced serum leptin levels in CD- and HFD-fed mice (Fig. 5n). Summarising these findings, nilotinib reduces nutrient storage and protects against HFD-induced obesity.

Nilotinib improves whole-body insulin sensitivity We performed GTTs and ITTs to assess whether nilotinib treatment improves insulin sensitivity. When mice were aged 16 weeks, the HFD caused glucose intolerance, while nilotinib treatment improved the phenotype (Fig. 6a). In line with the GTT results, 

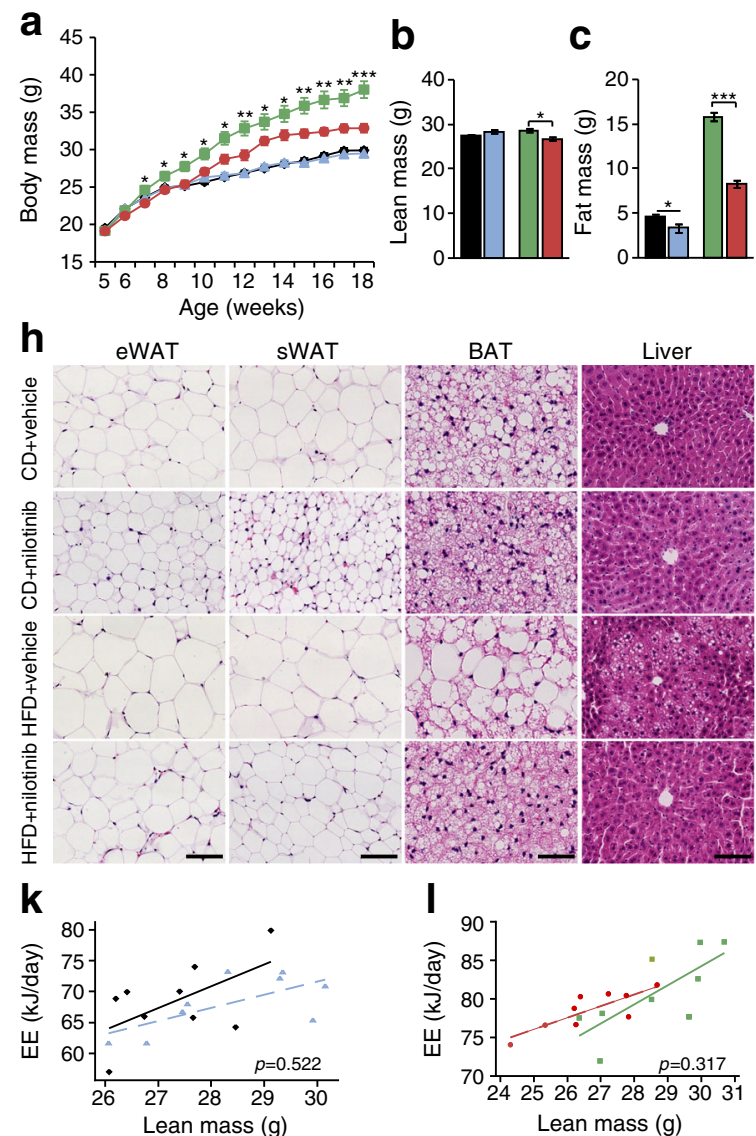

BAT
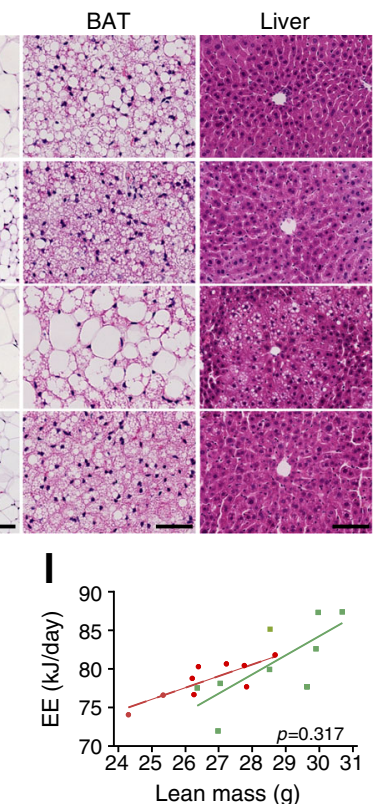

Fig. 5 Nilotinib protects against HFD-induced obesity. (a) Body mass of mice treated with nilotinib $(75 \mathrm{mg} / \mathrm{kg})$ or vehicle plus CD or HFD. CD+vehicle, $n=11$; CD+nilotinib, $n=12$; HFD+vehicle, $n=12$; HFD+ nilotinib, $n=12$. (b, c) Body lean (b) and fat (c) mass measured by MRI ( $n=9$ per group). $(\mathbf{d}-\mathbf{g})$ Mass of eWAT $(\mathbf{d}), \operatorname{sWAT}(\mathbf{e})$, BAT $(\mathbf{f})$ and liver (g). CD++vehicle, $n=11$; CD+nilotinib, $n=12$; HFD+vehicle, $n=11$; HFD+nilotinib, $n=11$. (h) Haematoxylin and eosin staining of eWAT, sWAT, BAT and liver. Scale bar, $100 \mu \mathrm{m}$ for eWAT, sWAT and liver or $50 \mu \mathrm{m}$ for BAT. (i, j) Oxygen consumption of indicated group of mice (i) and average oxygen consumption during light and dark period (j) $(n=9$ per group). (k, l) Statistical analysis of energy expenditure (EE) by GLM

ITT analysis revealed increased insulin sensitivity after nilotinib treatment under the HFD condition; no significant difference was found under the CD condition (Fig. 6b). Notably, after 16 weeks of HFD, there was no significant change in pancreatic morphology between control and nilotinib treatment (Fig. 6c). Further biochemical analysis showed that nilotinib treatment rescued HFD-reduced Akt phosphorylation only in sWAT and not in other tissues (Fig. 6d). This data indicates that c-Abl plays an important role in the regulation of subcutaneous fat insulin sensitivity under HFD challenge. Together, c-Abl kinase inhibition in subcutaneous fat could improve insulin sensitivity and contribute to weight reduction.

c-Abl inhibition improves insulin sensitivity by increasing adiponectin levels Multiple factors contribute to high-energy diet-induced insulin resistance, including inflammation,
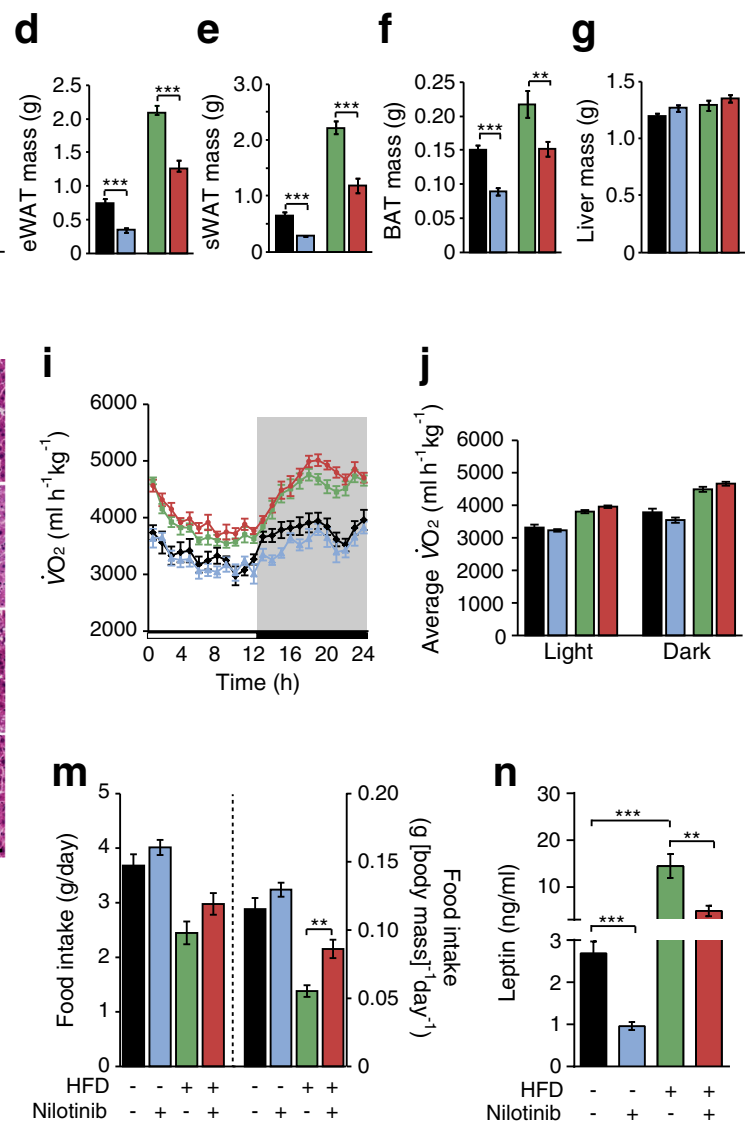

and ANCOVA of different groups ( $n=9$ per group). (m) Daily food intake in absolute values and normalised by body mass ( $n=9$ per group, $*^{*} p<0.01$ for indicated comparison; Student's $t$ test). (n) Leptin levels in serum ( $n=11$ per group). For graphs, data are means \pm SEM, except for (k) and (I). Black bars and diamonds, CD+vehicle; blue bars and triangles, CD+nilotinib; green bars and squares, HFD+vehicle; red bars and circles, HFD+nilotinib. $* p<0.05, * * p<0.01$ and $* * * p<0.001$ for indicated comparisons (ANOVA, unless indicated otherwise). In the line graphs in (a), $p$ values are for red circles vs green squares at the indicated time points

endoplasmic reticulum (ER) stress and adipokines [6, 30, 31]. To decipher the mechanism underlying the role of c-Abl in insulin resistance, we first studied whether c-Abl inhibition affected the inflammation in adipose tissues. Different types of adipose tissue confer different inflammation capability (e.g. there is a relatively higher immunological response in eWAT than in sWAT or BAT $[8,32,33])$. We found that the expression levels of F4/80 (also known as Adgre1), Mcpl and Pai-1 (also known as Serpine1) were markedly increased in eWAT under HFD challenge (ESM Fig. 2a-f). Compared with $c-A b l$ cKO (ESM Fig. 2a-c), nilotinib could significantly reduce the expression of F4/80, Mcpl and Pai-1 in eWAT, but not in sWAT or BAT, under the HFD condition (ESM Fig. 2d-f). The failure of c-Abl cKO (when compared with nilotinib) to inhibit HFD-induced inflammation might be due to low deletion efficiency of $c-A b l$ in eWAT when using the Fabp4-Cre 

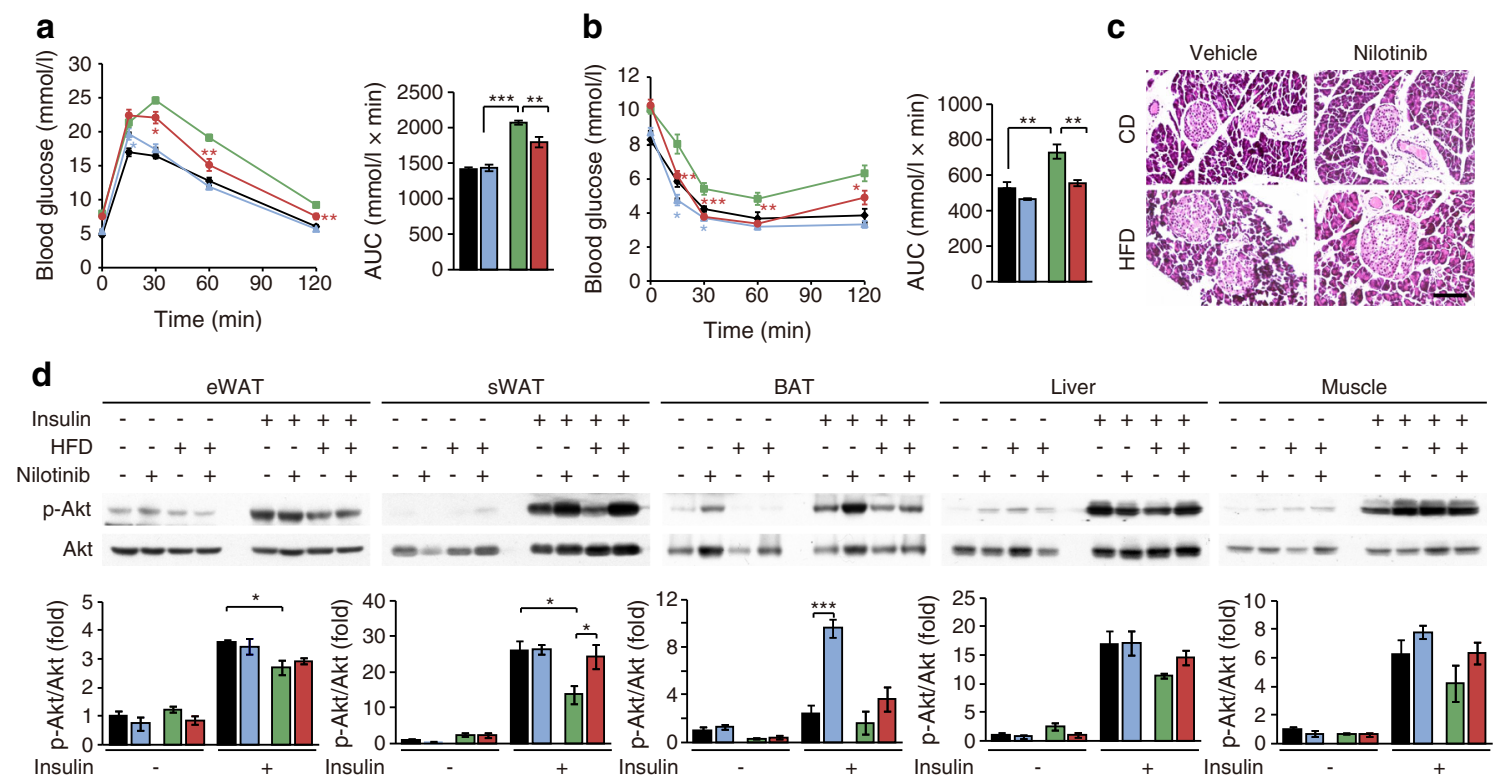

Fig. 6 c-Abl inhibition improves insulin sensitivity in vivo. (a) GTT analysis of nilotinib- $(75 \mathrm{mg} / \mathrm{kg})$ or vehicle-treated 16 -week-old mice fed with CD or HFD. The AUC for the GTT is also shown $(n=7$ or 8 per group). (b) ITT results for nilotinib- $(75 \mathrm{mg} / \mathrm{kg}$ ) or vehicle-treated mice fed with CD or HFD. The AUC for the ITT is also shown $(n=8$ per group). (c) Haematoxylin and eosin staining of pancreases from mice treated with nilotinib $(75 \mathrm{mg} / \mathrm{kg}$ ) or vehicle plus CD or HFD for 16 weeks. Scale bar, $100 \mu \mathrm{m}$. (d) Immunoblot of lysates from indicated tissues with

the antibodies indicated. Quantification of phosphorylated Akt in the indicated tissues is shown. For graphs, data are means \pm SEM. Black bars and diamonds, $\mathrm{CD}+$ vehicle; blue bars and triangles, $\mathrm{CD}+$ nilotinib; green bars and squares, HFD+vehicle; red bars and circles, HFD+nilotinib. $* p<0.05, * * p<0.01$ and $* * * p<0.001$ for indicated comparisons (ANOVA). In the line graphs in (a) and (b), $p$ values are for red circles vs green squares at the indicated time points

promoter (ESM Fig. 2a-c,g). The analysis of inflammation in different tissues suggests that the effect of Fabp4-Cre-mediated $c$ - $A b l$ deletion might be independent of inflammation.

JNK is the putative target kinase of inflammation factors and its phosphorylation/activation plays an important role in the development of inflammation-induced insulin resistance [34, 35]. We therefore examined the phosphorylation of JNK in adipose tissues from different models under HFD challenge. We found that HFD increased JNK phosphorylation in both eWAT and sWAT and that $c-A b l$ conditional deletion marginally inhibited JNK phosphorylation in sWAT but not in eWAT, which might be due to the lower efficiency of $c-A b l$ deletion in eWAT compared with sWAT (ESM Fig. 2g). Consistently, nilotinib significantly inhibited JNK1/2 phosphorylation in both sWAT and eWAT under the HFD condition (ESM Fig. 2h). However, our previous data show that both $c-A b l$ cKO and treatment with nilotinib improve insulin sensitivity in subcutaneous fat but not in eWAT or BAT (Figs 4h and 6d). Hence, we argue that the improvement in insulin sensitivity brought about by $c-A b l \mathrm{cKO}$ or inhibition might not be due to reduction in HFD-induced inflammation.

Recently, ER stress has emerged as a new player in obesity and type 2 diabetes and a considerable number of studies have highlighted its role in insulin resistance [36]. However, there was no significant difference in expression of Grp78, an ER stress marker, between controls and either $c-A b l$ cKO mice or nilotinib-treated mice under either CD or HFD challenge
(ESM Fig. 2g,h). These data indicated that ER stress does not contribute to c-Abl-induced insulin resistance.

Adipose tissues also secrete multiple adipokines, such as adiponectin and resistin, to regulate insulin sensitivity. For example, adiponectin increases insulin sensitivity and is mainly secreted from subcutaneous fat [37-39]. Two recent studies found that imatinib improves insulin sensitivity in the peripheral tissues via increasing plasma adiponectin levels [18, 19]. Consistent with this, we found that $\mathrm{c}-\mathrm{Abl} \mathrm{cKO}$ or $\mathrm{c}-\mathrm{Abl}$ inhibition significantly rescued HFD-induced adiponectin reduction (Fig. 7a,b). Resistin, mainly secreted from visceral fat, is associated with the development of insulin resistance and type 2 diabetes mellitus, [40,41]. We observed that HFD increased plasma resistin levels but that $c-A b l \mathrm{cKO}$ or nilotinib treatment failed to reduce its levels (Fig. 7c,d). Taken together, we argued that c-Abl inhibition might improve insulin sensitivity mainly through promoting adiponectin secretion from subcutaneous adipose tissue under HFD treatment (Fig. 7e). However, the detailed mechanism of c-Abl-regulated adiponectin needs further studied.

\section{Discussion}

In this work, we revealed a critical role for c-Abl in obesity through its regulation of insulin response. Genetic deletion of $c-A b l$ in adipose tissue or pharmacological inhibition of cAbl by nilotinib markedly attenuated HFD-induced obesity 

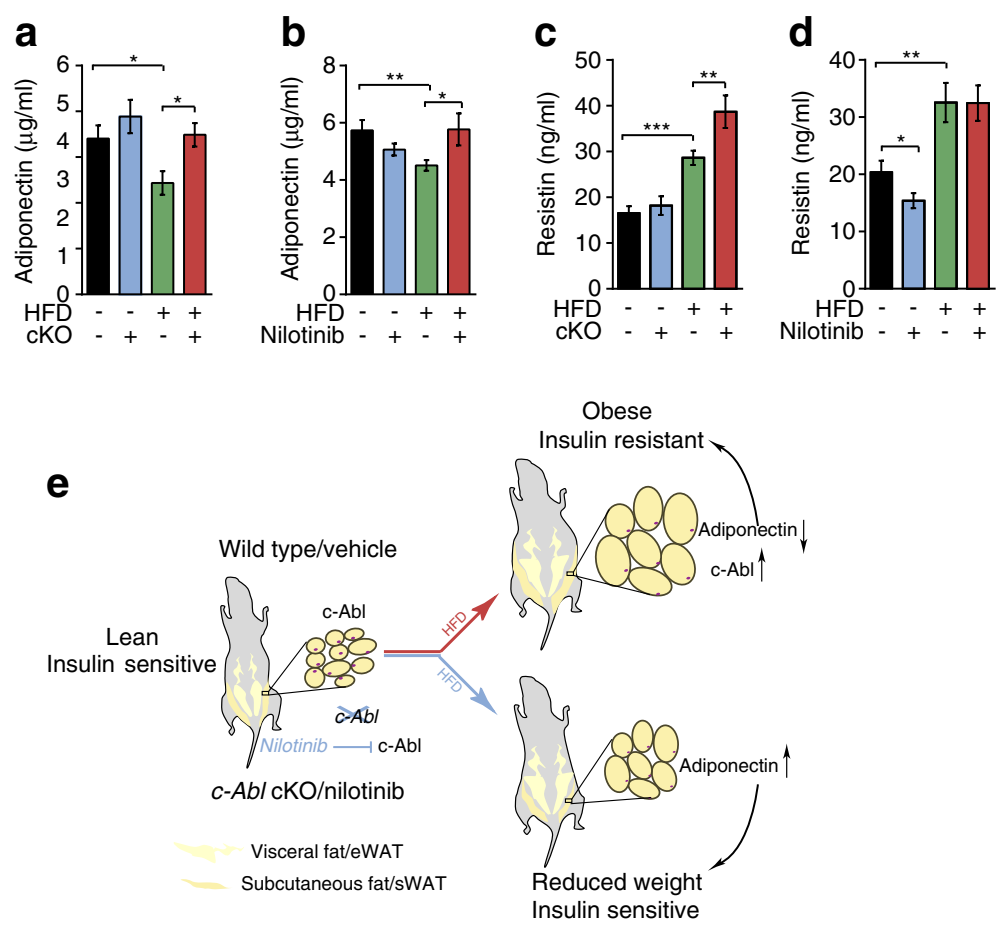

Fig. 7 c-Abl inhibition increases adiponectin levels in vivo. (a, c) Adiponectin (a) and resistin (c) levels in serum from WT and $c-A b l \mathrm{cKO}$ male mice fed with $\mathrm{CD}$ or HFD. Black bars, WT $+\mathrm{CD}, n=6$; blue bars, cKO+CD, $n=4$; green bars, WT+HFD, $n=16$; red bars, cKO+HFD, $n=10$. (b, d) Adiponectin (b) and resistin (d) levels in serum from nilotinib- $(75 \mathrm{mg} / \mathrm{kg})$ or vehicle-treated mice fed with CD or HFD. Black bars, CD+vehicle, $n=11$; blue bars, $\mathrm{CD}+$ nilotinib, $n=12$; green bars,

HFD+vehicle, $n=11$; red bars, HFD+nilotinib, $n=11$. $* p<0.05$, $* * p<0.01$ and $* * * p<0.001$ for indicated comparisons (ANOVA). (e) Model depicting the role of $\mathrm{c}-\mathrm{Abl}$ in obesity and obesity-associated insulin resistance. The red arrow represents the development of obesity/insulin resistance under HFD and the blue arrow represents the attenuation of detrimental effects of HFD on weight/insulin sensitivity via $c-A b l \mathrm{cKO}$ or c-Abl inhibition

and insulin resistance in vivo. Interestingly, we observed that both c-Abl specific deletion and nilotinib treatment reduced HFD-induced insulin resistance in subcutaneous fat through restoring the adiponectin level. Thus, our findings argue that subcutaneous c-Abl is important for whole-body insulin sensitivity and suggest c-Abl as a therapeutic target in the treatment of metabolic disorders.

White adipose tissue is distributed throughout the body and is divided into two major types: visceral fat and subcutaneous fat, each possessing unique cell-autonomous properties [8]. In contrast to visceral white adipose tissue, which is detrimental to metabolic homeostasis, sWAT is beneficial for metabolism by improving insulin sensitivity [8]. The expression levels of resistin and RBP-4 are high and there is a high distribution of macrophages, $\mathrm{T}$ cells and natural killer cells in visceral white adipose tissue [32, 40, 42]. Interestingly, there is a high expression level of adiponectin in subcutaneous fat [37-39]. In addition, it has been reported that the antilipolytic effect of insulin is greater in subcutaneous adipose tissue than in visceral adipose tissue [43], implying that subcutaneous adipose tissue might play a profound role in the regulation of insulin sensitivity and obesity. Interestingly, we found that c-Abl inhibition selectively increased the secretion of adiponectin in subcutaneous fat. This provides new evidence for the critical role of subcutaneous fat in maintaining whole-body insulin sensitivity.

The non-receptor tyrosine kinase c-Abl is activated by cellular stress, such as oxidative stress and DNA damage [44]. There is evidence showing that c-Abl plays a central role in CML and neurodegenerative diseases [12, 45]. Recently, extensive studies have linked c-Abl to islet beta cell survival and adipogenesis [20, 21, 23, 24]. By utilising genetic ablation and pharmacological inhibition, we found a new role for c-Abl in regulating diet-induced obesity and insulin resistance in vivo.

To study the role of c-Abl in adipose tissue, Fabp4-Cre was used to create adipose-specific deletion in vivo. Fabp4-Cre has been extensively used to target adipose tissue [25, 26]. However, recent studies showed that Fabp4-Cre lines lack tissue specificity $[26,46]$. We carefully examined the expression levels of c-Abl in metabolism-related tissues, including adipose tissue, liver, skeletal muscle and pancreas. We observed that Fabp4-Cre-mediated $c-A b l$ cKO indeed had low deletion efficiency in eWAT, sWAT, BAT and liver at the mRNA level, but at the protein level significant reduction of $\mathrm{c}-\mathrm{Abl}$ was only observed in sWAT (ESM Fig.1). In addition, $c-A b l$ cKO could not block HFD-induced eWAT mass gain and adipocyte size 
(Fig. 2d-h). Hence, Fabp4-Cre mediated $c$-Abl specific deletion might provide an ideal model with which to study the role of c-Abl in subcutaneous fat.

Notably, despite Fabp4-Cre having a low efficiency for deletion of $c-A b l$ in BAT, both genetic deletion of $c-A b l$ and pharmacological inhibition of c-Abl markedly reduced lipid accumulation in BAT (Figs $2 \mathrm{~h}, 5 \mathrm{~h}$ ). We examined features relating to BAT activation, including energy expenditure and Ucp1 expression. Unexpectedly, neither genetic deletion nor nilotinib treatment had any effect on these functions (Figs 3a-f and $5 \mathrm{i}-1)$. Hence, we argued that the reduced adipocyte size might be due to whole-body insulin sensitivity.

Last, we explored the mechanism by which $c$-Abl deletion or c-Abl inhibition improves insulin sensitivity. HFD is known to induce low-grade inflammation in adipose tissue [47] and cAbl inhibition has been shown to decrease the proinflammatory response in macrophages [48]. However, we found that $c-A b l$ specific deletion did not block HFD-induced inflammation in adipose tissue (ESM Fig. 2a-c), indicating that inflammation might not be the major factor involved in c-Ablmediated insulin resistance. We then sought to determine whether adipokines play a role in c-Abl-mediated insulin resistance. Interestingly, we found that adiponectin, mainly secreted from subcutaneous adipose tissue [37-39], was significantly increased by $c-A b l$ specific deletion or c-Abl inhibition under HFD challenge. However, there was no significant change in the level of resistin, which is mainly secreted from visceral fat $[40,41]$. Collectively, we propose that c-Abl promotes insulin resistance and obesity by regulating adiponectin secretion in subcutaneous fat in the presence of HFD challenge (Fig. 7e).

Taken together, our study highlights the important role of c$\mathrm{Abl}$ in obesity-associated insulin resistance. Inhibiting the activation of c-Abl or specifically reducing its expression in subcutaneous fat might be a promising therapeutic option for the treatment of obesity and related metabolic disorders.

Acknowledgements We thank the members of the Yuan laboratory for critical reading of the manuscript and helpful discussion.

Data availability All data supporting the findings of this study are available within the article and its ESM.

Funding This work was supported by the National Science Foundation of China (Grant no. 81630026, 81125010 and 81030025), the National Basic Research Program of China (973-2012CB910701 and 2013DFA31990) and Cross-disciplinary Collaborative Teams Program for Science, Technology and Innovation (2014-2016) from the Chinese Academy of Sciences.

Contribution statement RW and ZY designed the study and drafted the manuscript. RW, GN, WJ and ZY contributed to the study design and revised the article's intellectual content. RW and J-GS performed most of the experiments. J-QW helped to collect human tissue samples and acquired data. BL and QL helped to perform the nilotinib treatment of mice and acquired data. RW, J-GS, WJ and ZY analysed the data. J-GS, J-
QW, BL and QL revised the article and all authors approved the final version. $\mathrm{ZY}$ is responsible for the integrity of this work.

Duality of interest The authors declare that there is no duality of interest associated with this manuscript.

\section{References}

1. Ng M, Fleming T, Robinson M et al (2014) Global, regional, and national prevalence of overweight and obesity in children and adults during 1980-2013: a systematic analysis for the Global Burden of Disease Study 2013. Lancet 384:766-781

2. Lozano R, Naghavi M, Foreman K et al (2012) Global and regional mortality from 235 causes of death for 20 age groups in 1990 and 2010: a systematic analysis for the Global Burden of Disease Study 2010. Lancet 380:2095-2128

3. Zimmet P, Thomas CR (2003) Genotype, obesity and cardiovascular disease-has technical and social advancement outstripped evolution? J Intern Med 254:114-125

4. Saltiel AR, Kahn CR (2001) Insulin signalling and the regulation of glucose and lipid metabolism. Nature 414:799-806

5. Eckel RH, Grundy SM, Zimmet PZ (2005) The metabolic syndrome. Lancet 365:1415-1428

6. Kahn SE, Hull RL, Utzschneider KM (2006) Mechanisms linking obesity to insulin resistance and type 2 diabetes. Nature 444:840-846

7. Cnop M, Landchild MJ, Vidal J et al (2002) The concurrent accumulation of intra-abdominal and subcutaneous fat explains the association between insulin resistance and plasma leptin concentrations: distinct metabolic effects of two fat compartments. Diabetes 51:1005-1015

8. Tran TT, Kahn CR (2010) Transplantation of adipose tissue and stem cells: role in metabolism and disease. Nat Rev Endocrinol 6: 195-213

9. Sirvent A, Benistant C, Roche S (2008) Cytoplasmic signalling by the c-Abl tyrosine kinase in normal and cancer cells. Biol Cell 100: 617-631

10. Xiao L, Chen D, Hu P et al (2011) The c-Abl-MST1 signaling pathway mediates oxidative stress-induced neuronal cell death. J Neurosci 31: 9611-9619

11. Liu W, Wu J, Xiao L et al (2012) Regulation of neuronal cell death by c-Abl-Hippo/MST2 signaling pathway. PLoS One 7:e36562

12. Wu R, Chen $\mathrm{H}$, Ma J et al (2016) c-Abl-p38 $\alpha$ signaling plays an important role in MPTP-induced neuronal death. Cell Death Differ 23:542-552

13. Greuber EK, Smith-Pearson P, Wang J, Pendergast AM (2013) Role of ABL family kinases in cancer: from leukaemia to solid tumours. Nat Rev Cancer 13:559-571

14. Druker BJ, Sawyers CL, Kantariian H et al (2001) Activity of a specific inhibitor of the BCR-ABL tyrosine kinase in the blast crisis of chronic myeloid leukemia and acute lymphoblastic leukemia with the Philadelphia chromosome. N Engl J Med 344:1038-1042

15. Kantarjian H, Sawyers C, Hochhaus A et al (2002) Hematologic and cytogenetic responses to imatinib mesylate in chronic myelogenous leukemia. N Engl J Med 346:645-652

16. Barbany G, Hoglund M, Simonsson B, Swedish CMLG (2002) Complete molecular remission in chronic myelogenous leukemia after imatinib therapy. N Engl J Med 347:539-540

17. Veneri D, Franchini M, Bonora E (2005) Imatinib and regression of type 2 diabetes. N Engl J Med 352:1049-1050

18. Fitter S, Vandyke K, Schultz CG, White D, Hughes TP, Zannettino AC (2010) Plasma adiponectin levels are markedly elevated in imatinib-treated chronic myeloid leukemia (CML) patients: a 
mechanism for improved insulin sensitivity in type 2 diabetic CML patients? J Clin Endocrinol Metab 95:3763-3767

19. Hagerkvist R, Jansson L, Welsh N (2008) Imatinib mesylate improves insulin sensitivity and glucose disposal rates in rats fed a high-fat diet. Clin Sci (Lond) 114:65-71

20. Mokhtari D, Al-Amin A, Turpaev K et al (2013) Imatinib mesilateinduced phosphatidylinositol 3-kinase signalling and improved survival in insulin-producing cells: role of Src homology 2-containing inositol 5'-phosphatase interaction with c-Abl. Diabetologia 56:1327-1338

21. Xia CQ, Zhang P, Li S et al (2014) C-Abl inhibitor imatinib enhances insulin production by beta cells: c-Abl negatively regulates insulin production via interfering with the expression of $\mathrm{NKx} 2.2$ and GLUT-2. PLoS One 9:e97694

22. Fitter S, Vandyke K, Gronthos S, Zannettino AC (2012) Suppression of PDGF-induced PI3 kinase activity by imatinib promotes adipogenesis and adiponectin secretion. J Mol Endocrinol 48:229-240

23. Wilson B, Liotta LA, Petricoiniii E (2013) Dynamic protein pathway activation mapping of adipose-derived stem cell differentiation implicates novel regulators of adipocyte differentiation. Mol Cell Proteomics 12:2522-2535

24. Keshet R, Bryansker Kraitshtein Z, Shanzer M, Adler J, Reuven N, Shaul Y (2014) c-Abl tyrosine kinase promotes adipocyte differentiation by targeting PPAR-gamma 2. Proc Natl Acad Sci U S A 111: 16365-16370

25. He W, Barak Y, Hevener A et al (2003) Adipose-specific peroxisome proliferator-activated receptor gamma knockout causes insulin resistance in fat and liver but not in muscle. Proc Natl Acad Sci U S A 100:15712-15717

26. Jeffery E, Berry R, Church CD et al (2014) Characterization of Cre recombinase models for the study of adipose tissue. Adipocyte 3 : 206-211

27. Cannon B, Nedergaard J (2004) Brown adipose tissue: function and physiological significance. Physiol Rev 84:277-359

28. Arch JR, Hislop D, Wang SJ, Speakman JR (2006) Some mathematical and technical issues in the measurement and interpretation of open-circuit indirect calorimetry in small animals. Int J Obes 30: 1322-1331

29. Tschop MH, Speakman JR, Arch JR et al (2012) A guide to analysis of mouse energy metabolism. Nat Methods 9:57-63

30. Boden G, Cheung P, Kresge K, Homko C, Powers B, Ferrer L (2014) Insulin resistance is associated with diminished endoplasmic reticulum stress responses in adipose tissue of healthy and diabetic subjects. Diabetes 63:2977-2983

31. Han J, Murthy R, Wood B et al (2013) ER stress signalling through eIF $2 \alpha$ and CHOP, but not IRE1 $\alpha$, attenuates adipogenesis in mice. Diabetologia 56:911-924

32. O'Rourke RW, Metcalf MD, White AE et al (2009) Depot-specific differences in inflammatory mediators and a role for NK cells and
IFN-gamma in inflammation in human adipose tissue. Int J Obes 33:978-990

33. Bruun JM, Lihn AS, Pedersen SB, Richelsen B (2005) Monocyte chemoattractant protein-1 release is higher in visceral than subcutaneous human adipose tissue (AT): implication of macrophages resident in the AT. J Clin Endocrinol Metab 90:2282-2289

34. Wellen KE, Hotamisligil GS (2005) Inflammation, stress, and diabetes. J Clin Invest 115:1111-1119

35. Lee YH, Giraud J, Davis RJ, White MF (2003) c-Jun N-terminal kinase (JNK) mediates feedback inhibition of the insulin signaling cascade. J Biol Chem 278:2896-2902

36. Flamment M, Hajduch E, Ferre P, Foufelle F (2012) New insights into ER stress-induced insulin resistance. Trends Endocrinol Metab 23:381-390

37. Fujikawa R, Ito C, Nakashima R, Orita Y, Ohashi N (2008) Is there any association between subcutaneous adipose tissue area and plasma total and high molecular weight adiponectin levels? Metab Clin Exp 57:506-510

38. Nakamura Y, Sekikawa A, Kadowaki T et al (2009) Visceral and subcutaneous adiposity and adiponectin in middle-aged Japanese men: the ERA JUMP study. Obesity 17:1269-1273

39. Bluher M, Williams CJ, Kloting $\mathrm{N}$ et al (2007) Gene expression of adiponectin receptors in human visceral and subcutaneous adipose tissue is related to insulin resistance and metabolic parameters and is altered in response to physical training. Diabetes Care 30:3110 3115

40. Steppan CM, Lazar MA (2002) Resistin and obesity-associated insulin resistance. Trends Endocrinol Metab 13:18-23

41. McTernan PG, McTernan CL, Chetty R et al (2002) Increased resistin gene and protein expression in human abdominal adipose tissue. J Clin Endocrinol Metab 87:2407

42. Yang Q, Graham TE, Mody N et al (2005) Serum retinol binding protein 4 contributes to insulin resistance in obesity and type 2 diabetes. Nature 436:356-362

43. Meek SE, Nair KS, Jensen MD (1999) Insulin regulation of regional free fatty acid metabolism. Diabetes 48:10-14

44. Hantschel O, Superti-Furga G (2004) Regulation of the c-Abl and Bcr-Abl tyrosine kinases. Nat Rev Mol Cell Biol 5:33-44

45. Marley SB, Deininger MW, Davidson RJ, Goldman JM, Gordon MY (2000) The tyrosine kinase inhibitor STI571, like interferon- $\alpha$, preferentially reduces the capacity for amplification of granulocytemacrophage progenitors from patients with chronic myeloid leukemia. Exp Hematol 28:551-557

46. Lee KY, Russell SJ, Ussar S et al (2013) Lessons on conditional gene targeting in mouse adipose tissue. Diabetes 62:864-874

47. Chandalia M, Abate N (2007) Metabolic complications of obesity: inflated or inflamed? J Diabetes Complicat 21:128-136

48. Le Q, Daniel R, Chung SW et al (1998) Involvement of C-Abl tyrosine kinase in lipopolysaccharide-induced macrophage activation. J Immunol 160:3330-3336 